\title{
Efectividad biológica de insecticidas para el manejo de la resistencia del picudo del chile
}

Biological effectiveness of insecticides for resistance management of pepper weevil Eficácia biológica de inseticidas para o manejo da resistência de gorgulho pimenta

Fabián Avendaño Meza

Universidad Autónoma de Sinaloa, México

fabian@uas.edu.mx

\section{Resumen}

Se realizó un estudio de efectividad biológica de los insecticidas clorpirifos etil, malation, oxamil, thiametoxam y zcipermetrina para el control del picudo del chile en campos de La Cruz de Elota, El Rosario y Culiacán, Sinaloa. Los experimentos se establecieron en un diseño de bloques al azar con arreglo en parcelas divididas, con la variante 'Insecticida' en las parcelas chicas y 'Dosis' en el factor de parcelas grandes. Los resultados indican que la población de picudos de La Cruz de Elota presentó los niveles más bajos de control al registrar porcentajes inferiores al 50 \%; mientras que la población de El Rosario resultó ser más susceptible a los insecticidas evaluados, con porcentajes de efectividad superiores al $74 \%$. Todos los tratamientos insecticidas fueron diferentes estadísticamente al testigo.

Palabras clave: efectividad biológica, Capsicum annuum, Anthonomus eugenii, resistencia, insecticidas. 


\section{Abstract}

Is a study of biological effectiveness of the insecticides chlorpyrifos ethyl, malathion, oxamil, thiamentoxam and zcipermetrina for the control of pepper weevil in the fields of La Cruz de Elota, El Rosario and Culiacan, Sinaloa. Experiments were established in a randomized blocks design with arrangement of split plots, with the variant 'Insecticide' in the plots and 'Dose' in the factor of large plots. The results indicate that the population of weevils of La Cruz de Elota presented lower control levels, recording percentages below 50\%, while the population of El Rosario turned out to be more susceptible to insecticides evaluated, with percentages of effectiveness over $74 \%$. All insecticides treatments were statistically different from the control.

Key Words: biological effectiveness, Capsicum annuum, pepper weevil, Anthonomus eugenii, resistance, insecticides.

\section{Resumo}

Um estudo de eficácia biológica do inseticida clorpirifós etil, malathion, oxamil, thiamentoxam e zcipermetrina pelo controle do bicudo pimenta em campos de Elota La Cruz, El Rosario e Culiacan, Sinaloa foi realizada. Os experimentos foram estabelecidos em um delineamento em blocos casualizados dispostos em parcelas subdivididas, com a variante 'inseticida' em meninas e fator de 'doses' em lotes grandes parcelas. Os resultados indicam que a população gorgulho de La Cruz de Elota apresentaram os menores níveis de controle ao registar percentagens inferiores a 50\%; enquanto a população de El Rosario acabou por ser mais suscetíveis aos inseticidas avaliados, com percentuais acima de eficácia de 74\%. Todos os tratamentos inseticidas foram estatisticamente diferentes para a testemunha.

Palavras-chave: eficácia biológica, Capsicum annuum, Anthonomus eugenii, resistência, inseticidas.

Fecha Recepción: Junio 2016 Fecha Aceptación: Diciembre 2016 


\section{Introducción}

México es el principal exportador de chile (Capsicum annumm L.) para consumo en fresco en el mundo, con una superficie sembrada de 148968 ha, una producción de 2732635 t y una media nacional de $19 \mathrm{t} \mathrm{ha}^{-1}$ en el ciclo agrícola 2014. Los principales estados productores son Zacatecas, Chihuahua, San Luis Potosí y Sinaloa, con más de la mitad de la superficie sembrada en el país, se destaca este último por la producción alcanzada de 604773 t y un rendimiento de $50.44 \mathrm{t} \mathrm{ha}^{-1}$ (SIAP, 2016).

Entre las principales limitantes del cultivo de chile se encuentran las plagas insectiles, de éstas el picudo Anthonomus eugenii Cano se considera la plaga más importante y un problema clave durante las etapas de floración y fructificación en todas las zonas productoras (Jiménez, 2004; Toapanta et al., 2005; Rodríguez-Leyva et al., 2007). Se estima que en México se pierden de 70 a 80 millones de dólares anualmente sólo por el ataque del picudo (Rodríguez-Leyva et al., 2012).

Las prácticas que se utilizan para controlar al picudo del chile consisten de una combinación de control químico y cultural, lo cual mantiene las poblaciones de la plaga a niveles bajos, pero a raíz del uso intensivo de insecticidas, cada día se reduce el número de éstos capaces de ejercer un control satisfactorio, debido principalmente al desarrollo de resistencia (Riley y King, 1994; Servín et al., 2007). El manejo de esta plaga se había enfocado previamente en el uso de variedades tolerantes (Quiñonez y Lujan, 2002), recolección de frutos caídos (Capinera, 2002), trampeo masivo de adultos y uso de hongos entomopatógenos (Coudriet y Kishaba, 1988). Sin embargo, en la práctica el manejo del picudo del chile recae en aplicaciones de insecticidas químicos e inclusive existen en el mercado combinaciones de insecticidas de los grupos neonicotinoides con piretroides, los cuales han sido ampliamente aceptados por los productores (Ruíz et al., 2009) pero representan un riesgo potencial ya que generan una presión de selección extra sobre la plaga.

Una parte fundamental en el combate químico de plagas es el Manejo de Resistencia a Insecticidas (MRI) (FAO, 2012), que fue alentado por la creciente documentación de casos de artrópodos resistentes a plaguicidas (Georghiou y Lagunes, 1991) y el inicio de una base de datos interactiva del tema (Whalon et al., 2008). El MRI consiste en estrategias que tienen por objetivo conservar la susceptibilidad de los artrópodos plaga a los plaguicidas disponibles y mantener la 
efectividad de estos insumos, al prevenir o retrasar el desarrollo de razas resistentes para evitar el incremento de la dosis de campo requerida y, por tanto, no aumentar los costos de combate ni los riesgos a la salud o al ambiente (Lagunes-Tejeda et al., 2009).

El manejo adecuado del picudo del chile depende del monitoreo cuidadoso de las poblaciones de adultos y de su control mediante insecticidas, aplicados por la mañana o al atardecer cuando el insecto se encuentre expuesto en la superficie de la planta (Corrales, 2002). Al momento de seleccionar el insecticida, se deben utilizar productos de eficacia comprobada, ya que existen reportes que en el estado de Sinaloa, el picudo del chile mostró tolerancia a varios de ellos (Gastélum-Luque et al., 2004; Avendaño-Meza et al., 2010, 2014, 2016). Además, el control químico deberá estar sustentado en estudios de efectividad biológica de los insecticidas en el campo, lo que permitirá utilizar los que tengan mejor control de la plaga y evitar el uso de productos no efectivos y, con ello, el desarrollo de poblaciones resistentes y aplicaciones innecesarias que incrementan los riesgos de contaminación e intoxicación por plaguicidas y encarecen la producción (Lagunes-Tejeda et al., 2009). Atendiendo la problemática anterior, se planteó el presente trabajo con el objetivo de realizar estudios de efectividad biológica de los insecticidas clorpirifos etil y malation (fosforados), oxamil (carbamato), thiametoxam (neonicotinoide) y zcipermetrina (piretroide), comúnmente usados contra el picudo del chile en tres poblaciones del estado de Sinaloa, para llenar un vacío de información que se tiene respecto a este tópico y que sirva como una herramienta en la toma de decisiones para un manejo adecuado de la resistencia a insecticidas en poblaciones A. eugenii.

\section{Materiales y métodos}

Se realizaron tres experimentos en plantaciones comerciales de chile del estado de Sinaloa: uno

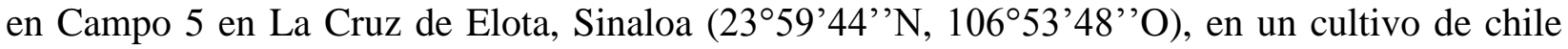
Bell para cosechar en verde y/o rojo establecido a campo abierto el 15 de septiembre de 2014;

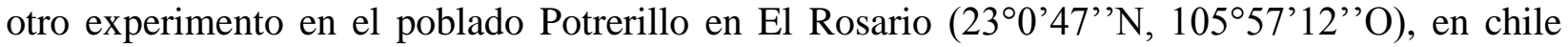
serrano a campo abierto el 3 de octubre de 2014 y un tercero en Agrícola Sabino en Culiacán (243' $31^{\prime}$ 'N $11^{\circ} 27^{\prime} 35^{\prime}$ '), en chile Bell establecido bajo malla sombra el 19 de septiembre de 2014. Se evaluaron las dosis bajas y altas recomendadas en la etiqueta de los insecticidas clorpirifos etil (Lorsban 75 WG, Dow Agrosciences), malation (Malation 1000, Indiapac), oxamil (Vydate L, DuPont), thiametoxam (Actara, Syngenta) y zcipermetrina (Mustang Max, FMC). Los 
tratamientos considerados fueron: clorpirifos etil $0.8 \mathrm{~kg} \mathrm{ha}^{-1}$, clorpirifos etil $1.2 \mathrm{~kg} \mathrm{ha}^{-1}$, malation 1.0 $\mathrm{L} \mathrm{ha}^{-1}$, malation $2.0 \mathrm{~L} \mathrm{ha}^{-1}$, oxamil $1.5 \mathrm{~L} \mathrm{ha}^{-1}$, oxamil $3.0 \mathrm{~L} \mathrm{ha}^{-1}$, thiametoxam $0.2 \mathrm{~kg} \mathrm{ha}^{-1}$, thiametoxam $0.4 \mathrm{~kg} \mathrm{ha}^{-1}$, zcipermetrina $0.3 \mathrm{~L} \mathrm{ha}^{-1}$ y zcipermetrina $0.6 \mathrm{~L} \mathrm{ha}^{-1}$, en un diseño de bloques al azar con arreglo en parcelas divididas, distribuidos en dos parcelas grandes con las variantes de dosis bajas y altas, comparados con un testigo donde no se aplicó insecticida, para una suma de seis factores de variación y un total de 12 tratamientos, los cuales se replicaron cuatro veces. Se realizaron cuatro aplicaciones de cada insecticida los días 3, 10, 17 y 24 de marzo de 2015 en La Cruz de Elota; los días 4, 11, 18 y 25 de marzo de 2015 en El Rosario y los días 1, 6, 12 y 16 de mayo de 2015 en Culiacán. Las aplicaciones se realizaron con una bomba de mochila motorizada calibrada para asperjar 300 litros de agua por hectárea. El parámetro de evaluación fue el promedio de adultos contados en 30 terminales por unidad experimental (se revisaron visualmente botones florales, flores y yemas terminales), los muestreos se realizaron al siguiente día de la aplicación entre las 7:00 y 10:00 am cuando la mayoría de los picudos se están alimentando (Corrales, 2002). El porcentaje de efectividad se calculó con el promedio de los adultos de picudo en cada tratamiento para cada insecticida, mediante la fórmula de Abbott (Abbott, 1925). El análisis estadístico se realizó con el programa SAS ${ }^{\circledR}$ para Windows ${ }^{\circledR}$ Ver. 9.3 (SAS Institute Inc, 2011), la comparación de medias se hizo con la prueba de Tukey con $5 \%$ de significancia.

\section{Resultados}

El análisis de los resultados obtenidos en La Cruz de Elota, Sinaloa indica que existe diferencia significativa en la interacción entre los insecticidas con las dosis; así como entre los tratamientos del factor insecticidas, no se encontró diferencia entre los promedios de adultos registrados en las factor 'Dosis'. La comparación de medias realizada con la interacción de los insecticidas con las dosis altas y bajas muestra diferencias entre estos y el testigo (cuadro 1). En todos los tratamientos se registraron valores entre 4.28 y 4.72 adultos en promedio y no son estadísticamente diferentes entre sí, pero sí difieren del testigo. El porcentaje de participación de los insecticidas fue relativamente bajo en esta región, ya que sólo aportaron entre 42.37 y 47.71 $\%$ del control de la plaga, considerando los promedios de las dosis bajas y altas y sólo cuatro tratamientos (malation a dosis baja, clorpirifos etil a dosis alta, zcipermetrina a dosis baja y oxamil a dosis alta) lograron superar del $50 \%$ de control (figura 1). 
Cuadro 1. Promedio de adultos de picudo del chile Anthonomus eugenii Cano en cuatro muestreos realizados en La Cruz de Elota, Sinaloa, 2015.

\begin{tabular}{|c|c|c|c|c|c|c|c|c|c|}
\hline \multicolumn{4}{|c|}{ Interacción Dosis x Insecticida } & \multicolumn{3}{|l|}{ Insecticida } & \multicolumn{3}{|l|}{ Dosis } \\
\hline Tratamiento & Dosis & $\mathrm{N}$ & Media* & Tratamiento & $\mathrm{N}$ & Media* & Tratamiento & $\mathrm{N}$ & Media \\
\hline Testigo & Alta & 4 & $8.19 \mathrm{a}$ & Testigo & 8 & $8.19 \mathrm{a}$ & Baja & 24 & 5.32 \\
\hline Testigo & Baja & 4 & $8.19 \mathrm{a}$ & Clorpirifos & 8 & $4.72 \mathrm{~b}$ & Alta & 24 & 5.00 \\
\hline Oxamil & Baja & 4 & $5.56 \mathrm{~b}$ & Oxamil & 8 & $4.69 \mathrm{~b}$ & & & \\
\hline Clorpirifos & Baja & 4 & $5.50 \mathrm{~b}$ & Thiametoxam & 8 & $4.69 \mathrm{~b}$ & & & \\
\hline Malation & Alta & 4 & $4.75 \mathrm{~b}$ & Malation & 8 & $4.41 \mathrm{~b}$ & & & \\
\hline Thiametoxam & Baja & 4 & $4.69 \mathrm{~b}$ & Zcipermetrina & 8 & $4.28 \mathrm{~b}$ & & & \\
\hline Thiametoxam & Alta & 4 & $4.69 \mathrm{~b}$ & & & & & & \\
\hline Zcipermetrina & Alta & 4 & $4.62 \mathrm{~b}$ & & & & & & \\
\hline Malation & Baja & 4 & $4.06 \mathrm{~b}$ & & & & & & \\
\hline Clorpirifos & Alta & 4 & $3.94 \mathrm{~b}$ & & & & & & \\
\hline Zcipermetrina & Baja & 4 & $3.94 \mathrm{~b}$ & & & & & & \\
\hline Oxamil & Alta & 4 & $3.81 \mathrm{~b}$ & & & & & & \\
\hline
\end{tabular}

*Medias con la misma letra son estadísticamente iguales, según Tukey $\alpha=0.05$

Baja $=$ Dosis bajas de insecticida en la parcela grande

Alta $=$ Dosis altas de insecticida en la parcela grande

$\mathrm{N}=$ Número de observaciones

Fuente: elaboración propia.

Figura 1. Porcentajes de efectividad de cinco insecticidas en la población de picudo del chile $A$. eugenii de La Cruz de Elota, Sinaloa. 2015.

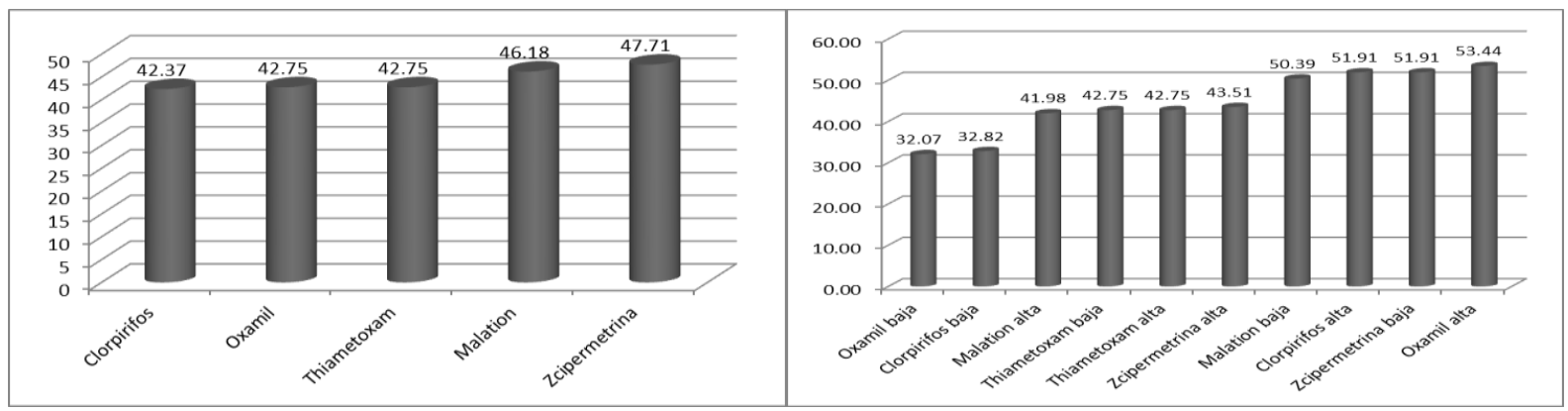

Fuente: elaboración propia.

El análisis de varianza de los datos del experimento realizado en El Rosario, Sinaloa indica que existen diferencias significativas entre los tratamientos en la interacción dosis con insecticidas y en el factor 'Insecticida'; no existe diferencia entre los componentes del factor parcelas grandes (dosis bajas y altas). La comparación de medias realizada con la interacción de las dosis altas y bajas y los insecticidas ubicados en la parcela chica, muestra diferencias entre los tratamientos y ubica como el más efectivo al clorpirifos etil a dosis alta, ya que en los cuatro muestreos no se registró un solo insecto (cuadro 2). La prueba de comparación de medias de Tukey $(\alpha=0.05)$ 
realizada a los insecticidas con el promedio de ambas dosis separa a los tratamientos en dos grupos; todos los tratamientos registraron valores entre 0.31 y 0.72 adultos en promedio y no son estadísticamente diferentes entre sí; sin embargo, difieren del testigo que registró un promedio de 2.81 adultos de picudos del chile. En esta región, a diferencia de la anterior, se observó una efectividad superior al $74 \%$ en el control de la plaga por parte de todos los insecticidas (figura 2). Lo anterior debido a que la plaga en esta región ha estado poco sometida a la presión de selección por insecticidas, lo que se refleja en una efectividad superior de los mismos y se constituye, por el momento, como un reservorio de individuos susceptibles para el manejo de la resistencia a esta plaga.

Cuadro 2. Promedio de adultos de picudo del chile Anthonomus eugenii Cano en cuatro muestreos realizados en El Rosario, Sinaloa, 2015.

\begin{tabular}{|c|c|c|c|c|c|c|c|c|c|}
\hline \multicolumn{4}{|c|}{ Interacción Dosis x Insecticida } & \multicolumn{3}{|l|}{ Insecticida } & \multicolumn{3}{|l|}{ Dosis } \\
\hline Tratamiento & Dosis & $\mathrm{N}$ & Media* & Tratamiento & $\mathrm{N}$ & Media* & Tratamiento & $\mathrm{N}$ & Media \\
\hline Testigo & Baja & 4 & $2.81 \mathrm{a}$ & Testigo & 8 & $2.81 \mathrm{a}$ & Baja & 24 & 0.94 \\
\hline Testigo & Alta & 4 & $2.81 \mathrm{a}$ & Thiametoxam & 8 & $0.72 \mathrm{~b}$ & Alta & 24 & 0.84 \\
\hline Thiametoxam & Alta & 4 & $1.12 \mathrm{~b}$ & Malation & 8 & $0.59 \mathrm{~b}$ & & & \\
\hline Clorpirifos & Baja & 4 & $0.81 \mathrm{bc}$ & Oxamil & 8 & $0.50 \mathrm{~b}$ & & & \\
\hline Oxamil & Baja & 4 & $0.75 \mathrm{bc}$ & Clorpirifos & 8 & $0.41 \mathrm{~b}$ & & & \\
\hline Malation & Baja & 4 & $0.62 \mathrm{bcd}$ & Zcipermetrina & 8 & $0.31 \mathrm{~b}$ & & & \\
\hline Malation & Alta & 4 & $0.56 \mathrm{bcd}$ & & & & & & \\
\hline Thiametoxam & Baja & 4 & $0.31 \mathrm{~cd}$ & & & & & & \\
\hline Zcipermetrina & Alta & 4 & $0.31 \mathrm{~cd}$ & & & & & & \\
\hline Zcipermetrina & Baja & 4 & $0.31 \mathrm{~cd}$ & & & & & & \\
\hline Oxamil & Alta & 4 & $0.25 \mathrm{~cd}$ & & & & & & \\
\hline Clorpirifos & Alta & 4 & 0.00 & & & & & & \\
\hline
\end{tabular}

*Medias con la misma letra son estadísticamente iguales, según Tukey $\alpha=0.05$

Baja $=$ Dosis bajas de insecticida en la parcela grande

Alta $=$ Dosis altas de insecticida en la parcela grande

$\mathrm{N}=$ Número de observaciones

Fuente: elaboración propia. 
Figura 2. Porcentajes de efectividad de cinco insecticidas en la población de picudo del chile $A$. eugenii de El Rosario, Sinaloa. 2015.

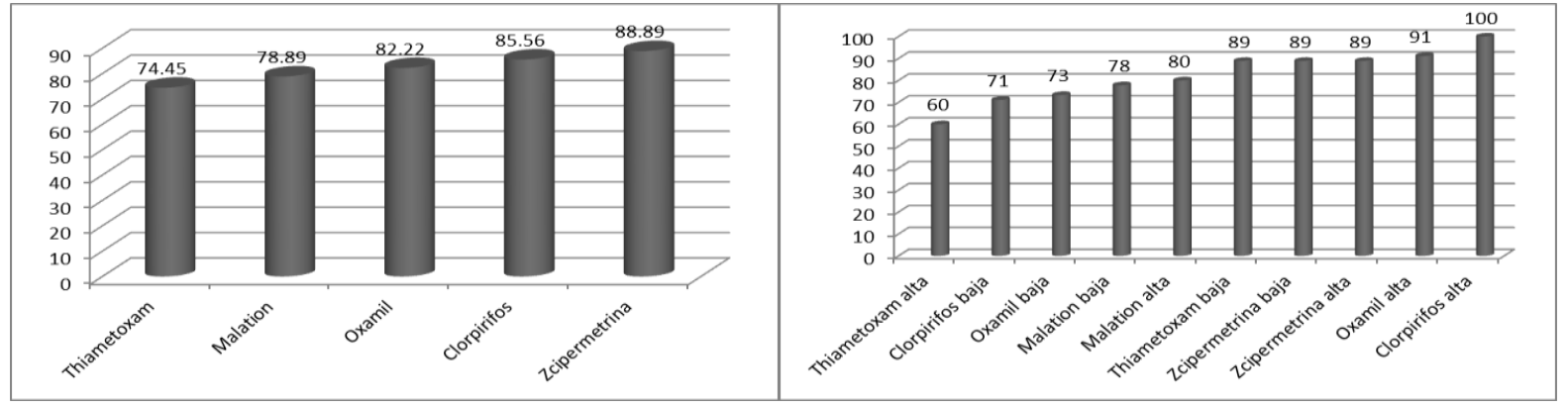

Fuente: elaboración propia.

En el experimento realizado en Culiacán, en cultivo bajo malla sombra se presentó una respuesta intermedia entre las poblaciones de La Cruz y El Rosario. El análisis de varianza realizado con los datos de la interacción dosis $\mathrm{x}$ insecticida muestra diferencia significativa entre los tratamientos, también se registraron diferencias entre los eventos del factor 'Insecticida', no hubo diferencia estadística con el factor 'Dosis'. El mayor número de insectos se registró en el testigo y fue diferente significativamente $(\mathrm{p} \leq 0.05)$ de los tratamientos insecticidas, de éstos se observa una mayor eficacia en el tratamiento con oxamil al registrar el promedio más bajo de insectos (cuadro 3) y el mejor porcentaje de control (figura 3), este tratamiento fue estadísticamente similar a thiametoxam y zcipermetrina y diferente a malation y clorpirifos etil. La prueba de medias de la interacción dosis-insecticidas separa al testigo de los demás tratamientos, los cuales tuvieron el mismo nivel de significancia estadística. Los porcentajes de control fluctuaron entre 56 y $80 \%$ para el factor insecticidas y entre 52 y $84 \%$. 
Cuadro 3. Promedio de adultos de picudo del chile Anthonomus eugenii Cano en cuatro muestreos en cultivo en malla sombra realizados en Culiacán, Sinaloa, 2015.

\begin{tabular}{|c|c|c|c|c|c|c|c|c|c|c|}
\hline \multicolumn{4}{|c|}{ Interacción Dosis x Insecticida } & \multicolumn{4}{|l|}{ Insecticida } & \multicolumn{3}{|l|}{ Dosis } \\
\hline Tratamiento & Dosis & $\mathrm{N}$ & Media* & Tratamiento & $\mathrm{N}$ & Media & & Tratamiento & $\mathrm{N}$ & Media \\
\hline Testigo & Alta & 4 & $8.00 \mathrm{a}$ & Testigo & 8 & 8.00 & $\mathrm{a}$ & Baja & 24 & 3.85 \\
\hline Testigo & Baja & 4 & $8.00 \mathrm{a}$ & Malation & 8 & 3.47 & $\mathrm{~b}$ & Alta & 24 & 3.36 \\
\hline Malation & Baja & 4 & $3.81 \mathrm{~b}$ & Clorpirifos & 8 & 3.31 & $\mathrm{~b}$ & & & \\
\hline Clorpirifos & Baja & 4 & $3.69 \mathrm{~b}$ & Thiametoxam & 8 & 2.69 & bc & & & \\
\hline Zcipermetrina & Baja & 4 & $3.25 \mathrm{~b}$ & Zcipermetrina & 8 & 2.66 & $\mathrm{bc}$ & & & \\
\hline Thiametoxam & Baja & 4 & $3.06 \mathrm{~b}$ & Oxamil & 8 & 1.53 & $\mathrm{c}$ & & & \\
\hline Clorpirifos & Alta & 4 & $2.81 \mathrm{~b}$ & & & & & & & \\
\hline Malation & Alta & 4 & $2.75 \mathrm{~b}$ & & & & & & & \\
\hline Thiametoxam & Alta & 4 & $2.56 \mathrm{~b}$ & & & & & & & \\
\hline Zcipermetrina & Alta & 4 & $2.31 \mathrm{~b}$ & & & & & & & \\
\hline Oxamil & Baja & 4 & $1.81 \mathrm{~b}$ & & & & & & & \\
\hline Oxamil & Alta & 4 & $1.25 \mathrm{~b}$ & & & & & & & \\
\hline
\end{tabular}

*Medias con la misma letra son estadísticamente iguales, según Tukey $\alpha=0.05$

Baja $=$ Dosis bajas de insecticida en la parcela grande

Alta $=$ Dosis altas de insecticida en la parcela grande

$\mathrm{N}=$ Número de observaciones

Fuente: elaboración propia.

Figura 3. Porcentajes de efectividad de cinco insecticidas en la población de picudo del chile $A$. eugenii de Culiacán, Sinaloa. 2015.

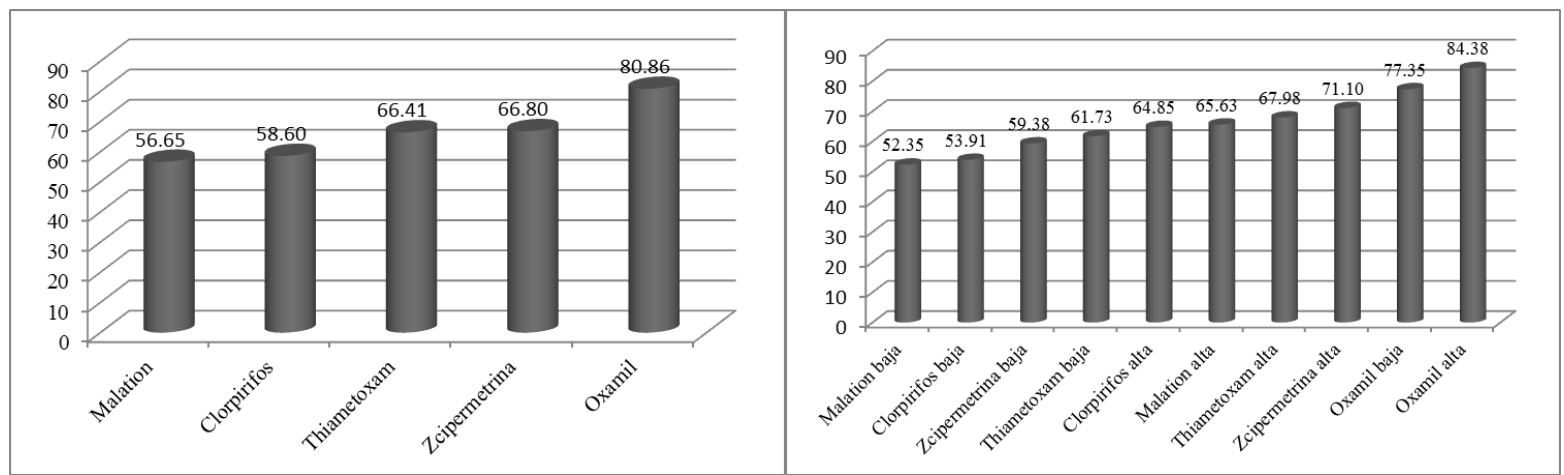

Fuente: elaboración propia. 


\section{Discusión}

En la población de La Cruz de Elota se presentaron los porcentajes de control más bajos, ya que la eficacia de los insecticidas apenas superó $50 \%$ con malation a dosis baja, y clorpirifos, zcipermetrina y oxamil a dosis alta. Lo anterior está relacionado con un aumento en la tolerancia de la población de A. eugenii a los insecticidas usados para su combate, principalmente los incluidos en el presente estudio (Avendaño-Meza et al., 2010, 2016). En esta zona no se observó diferencia significativa entre las dosis bajas y altas para todos los insecticidas, lo que hace innecesario el aumento de dosis cuando se incrementa la población del insecto. Además, al limitar el uso de estos insecticidas, se prioriza el uso de técnicas de manejo de la plaga alternativas al uso de agroquímicos, como el control legal y cultural. Es importante respetar y, si es posible, incrementar el tiempo de la ventana fitosanitaria libre del cultivo, así como un programa riguroso de monitoreo y recolección manual de adultos para disminuir la presión de selección sobre la plaga y favorecer un manejo adecuado de la resistencia, tal como lo sugieren Lagunes-Tejeda et al. (2009). Corrales (2002) realizó un trabajo en La Cruz de Elota, Sinaloa para comparar el control químico convencional y una estrategia biorracional contra picudo del chile; señala que la mayor eficacia se obtuvo con la aplicación de insecticidas químicos, no sin antes ejercer una enorme presión de selección sobre la plaga, ya que se realizaron 27 aplicaciones para mantener a este insecto por debajo del umbral económico.

Los promedios más bajos en la cantidad de adultos de picudo se observaron en la población de El Rosario, Sinaloa, los porcentajes de control oscilaron entre 74 y $89 \%$ para los insecticidas evaluados, independientemente de la dosis aplicada. Lo anterior permite reconocer que con las dosis bajas se logrará un control satisfactorio de la plaga y un aumento de éstas no se traduce necesariamente en una mejora de la eficacia del producto, permitiendo con ello un manejo racional de los insumos utilizados y la conservación de la susceptibilidad del picudo a los insecticidas. El objetivo del manejo de la resistencia es prevenir o retardar la acumulación de individuos resistentes en las poblaciones de plagas, de manera que se preserve la efectividad de los plaguicidas disponibles. El manejo de la resistencia puede también llamarse manejo de la susceptibilidad como medio de mantener un alto porcentaje de genes susceptibles dentro de la población de la plaga y así mantener los genes de resistencia al mínimo, tal como lo sugieren investigadores de la FAO (2012). 
En la población de insectos de Culiacán hubo una respuesta intermedia entre las tres poblaciones, los tratamientos malation y clorpirifos etil (dosis bajas) tuvieron una respuesta parecida a la obtenida en La Cruz de Elota, con efectividad de 52.35 y $53.91 \%$, respectivamente. Mientras que en el tratamiento a base de oxamil se lograron porcentajes de efectividad superiores al $77 \%$, resultados congruentes a los obtenidos por Seal y Schster (1995) y relacionados con bioensayos de laboratorio de estudios de susceptibilidad a insecticidas efectuados por Avendaño-Meza et al., (2010, 2014). La población de picudos de esta zona está respondiendo a la presión de selección de los insectidas zcipermetrina y thiametoxam, ya que sólo se lograron controles satisfactorios utilizando las dosis altas de los insecticidas. Estas fallas en el control se relacionan con el incremento en los niveles de tolerancia a los mismos, ya que los productos antes señalados se aplican intensivamente y en los momentos críticos de ataque de esta plaga, por lo que la presión de selección continúa incrementándose y dificulta el retorno a la susceptibilidad en un corto a mediano plazo. Gutiérrez-Olivares et al. (2007) han documentado la inestabilidad de la resistencia de insecticidas neonicotinoides; en condiciones de laboratorio decreció de 6.9 a 2.8x para thiametoxam en cuatro generaciones libres de presión de selección, por ello la importancia de aplicar este tipo de insecticidas sólo en los periodos críticos del desarrollo del cultivo. A su vez, García-Nevarez (2012) evaluaron la eficacia de insecticidas biorracionales y convencionales sobre picudo del chile en Chihuahua y encontraron que thiametoxam, clorpirifos etil y lamdacihalotrina mostraron un impacto en la reducción de la población de esta plaga hasta cinco días después de la aplicación, el tratamiento biorracional se comportó igual que el testigo. Por su parte, Ruiz et al. (2009) concluyen que la azadiractina es una buena alternativa en el control de $A$. eugenii, y podría sustituir el uso de oxamil y neonicotioides cuando las poblaciones de la plaga no son altas, minimizando de esta manera la presión de selección para resistencia a estos plaguicidas. En un trabajo realizado por Addesso et al. (2014) para investigar el efecto de los productos de bajo riesgo (arcilla de caolín, tierra de diatomeas, neem y extracto de Chenopodium ambrosioides) contra picudo del chile, comparados con los insecticidas thiametoxam y oxamil aplicados en rotación y un testigo no tratado, encontraron que el único tratamiento donde se incrementó el rendimiento fue en la rotación de insecticidas convencionales. Por otro lado, con los productos orgánicos no se incrementó significativamente el rendimiento, pero su uso disminuyó el daño total, lo que indica su posible utilidad en combinación con plaguicidas 
químicos convencionales o de bajo impacto ambiental bajo una estrategia de manejo integrado de plagas.

\section{Conclusiones}

La población de picudo del chile de La Cruz de Elota se mostró tolerante a los insecticidas clorpirifos etil, malation, oxamil, thiametoxam y zcipermetrina, con bajos niveles de control.

La población de insectos de Culiacán está respondiendo a la presión de selección ejercida por las aplicaciones de insecticidas, principalmente los organofosforados malation y clorpirifos etil, y el neonicotinoide thiametoxam, ya que se observó baja eficacia en ambas dosis (bajas y altas). Con zcipermetrina se obtuvo buena eficacia sólo a dosis alta, mientras que con oxamil se observó buena eficacia aún con la dosis baja.

En la población de picudos de El Rosario, todos los insecticidas evaluados presentaron buenos porcentajes de control, por lo que se considera una población susceptible y un reservorio de genes de susceptibilidad para esta plaga.

En las tres zonas en estudio no se observó diferencia significativa entre las dosis altas y bajas de los insecticidas, por lo que de ser necesario aplicar uno de estos insumos es recomendable aplicarlos a dosis baja para reducir costos y retardar el desarrollo de resistencia, y mantener con ello la eficacia de los productos por un periodo mayor de tiempo. 


\section{Bibliografía}

Abbott, W.S. (1925). A method of computing the effectiveness of an insecticide. J. Econ. Entomol, 18, 265-267.

Addesso, K.M., Stansly, P.A., Kostyk, B.C. y McAuslane, H.J. (2014). Organic treatments for control of pepper weevil (Coleoptera: Curculionidae). Florida Entomologist, 97(3), 11481156. DOI: http://dx.doi.org/10.1653/024.097.0322

Avendaño-Meza, F., Corrales-Madrid, J.L., Parra-Terraza, S., Medina-López, R., GasparAguilar, S.S. y Avendaño-Jatomea, F.D. (2016). Líneas base de susceptibilidad a tres insecticidas en poblaciones de picudos del chile Anthonomus eugenii cano, 1894 (coleóptera: curculionidae) del estado de Sinaloa. Entomología Mexicana, 3, 775-780.

Avendaño-Meza, F., López-Meza, M., Gastélum-Luque, R., Medina-López, R., Godoy-Angulo, T.P. y Gaspar-Aguilar, S.S. (2010). Susceptibilidad a insecticidas en poblaciones de picudo del chile Anthonomus eugenii Cano en La Cruz de Elota, Sinaloa. Entomología Mexicana, 9, 711-716.

Avendaño-Meza, F., Parra-Terraza, S., Gastélum-Luque, R., López-Meza, M., Medina-López, R. y Yáñez-Juárez, M.G. (2014). Líneas base de resistencia a cinco insecticidas en poblaciones de picudo del chile Anthonomus eugenii Cano de Culiacán, Sinaloa. Entomología Mexicana, 1, 834-839.

Capinera, J.L. (2002). Pepper weevil Anthonomus eugenii Cano (Insecta: Coleóptera: Curculionidae). Document EENY-278. Florida. Cooperative Extensión Service. Institute of Food and Agricultural Science. University of Florida, E.U., 6 p.

Corrales, M.J.L. (2002). Estrategias biorracionales para el manejo de las principales plagas del cultivo de chile en La Cruz de Elota, Sinaloa. Tesis de Doctorado. Instituto de Fitosanidad. Colegio de Postgraduados. Montecillo, Edo. México, 113 p.

Coudriet, D.L. \& Kishaba, A.N. (1988). Bioassay Procedure for an Attractant of the Pepper Weevil (Coleoptera: Curculionidae). Journal of Economic Entomology, 81(5), 1499-1502. 
FAO (2012). Código internacional de conducta para la distribución y utilización de plaguicidas. Directrices sobre la prevención y manejo de la resistencia a los plaguicidas. Recuperado el 03 de agosto de 2016, de www.fao.org/fileadmin/templates/agphome/documents/ Pests_Pesticides/Code/FAO_RMG_SP.pdf

García-Nevarez, G., Campos-Figueroa, M., Chávez-Sánchez, N. \& Quiñonez-Pando, F.J. (2012). Efficacy of biorational and conventional insecticides against the pepper weevil, Anthonomus eugenii Cano (Coleoptera: Curculionidae) in the South-Central Chihuahua. Southwestern Entomologist, 37(3), 391-401.

Gastélum-Luque, R., Avendaño-Meza, F., Rodríguez-Vázquez, J.F., López-Meza, M. \& GodoyAngulo, T.P. (2004). Tolerancia a insecticidas en 'picudo del chile' Anthonomus eugenii Cano, procedentes de la Cruz de Elota, Sinaloa. Entomología Mexicana, 3, 728-731.

Georghiou, G.P. \& Lagunes, A. (1991). The occurrence of resistance to pesticides in arthropods. Food and Agriculture Organization of the United Nations. Rome, 287 p.

Gutiérrez-Olivares, M., Rodríguez-Maciel, J.C., Llanderal-Cázares, C., Terán-Vargas, A.P., Lagunes-Tejeda, A. \& Díaz-Gómez, O. (2007). Estabilidad de la resistencia a neonicotinoides en Bemisia tabaci (Gennadius), biotipo B de San Luis Potosí, México. Agrociencia, 41, 913-920.

Jiménez, S. H. (2004). Guía para el manejo integrado de plagas del cultivo de chile dulce. Centro Agronómico Tropical de Investigación y enseñanza CATIE. Proyecto Manejo Integrado de Plagas. Turrialba, Costa Rica, 141 p.

Lagunes-Tejeda, A, Rodríguez-Maciel, J.C. y De Loera-Barocio, J.C. (2009). Susceptibilidad a insecticidas en poblaciones de artrópodos de México. Agrociencia, 43(2), 173-196.

Quiñones, F. \& Luján, M. (2002). Differential response of jalapeño genotypes to the damage for pepper weevil Anthonomus eugenii Cano (Coleoptera: Curculionidae). Proceedings of the 16th International Pepper Conference. Tamaulipas, México del 10 al 12 de noviembre del 2002 
Riley, D.G. \& King, E.G. (1994). Biology and management of pepper weevil Anthonomus eugenii Cano (Coleoptera: Curculionidae): a review. Trends Agricultural Science, 2, 109121.

Rodríguez-Leyva, E., Lomelí-Flores, J.R., Valdez-Carrasco, J.M., Jones, R.W. \& Stansly, P.A. (2012). New Records of species and locations of parasitoids of the pepper weevil in Mexico. Southwestern Entomologist, 37(1), 73-83.

Rodríguez-Leyva, E., Stansly, P.A. Schuster, D.J. \& Bravo-Mosqueda, E. (2007). Diversity and distribution of parasitoids of Anthonomus eugenii (Coleóptera: Curculionidae) from Mexico and prospects for biological control. Florida Entomologist, 90, 693-702.

Ruíz, S.E., Aguilar, O., Cristóbal, A.J., Tun, S., Latournerie, M.L. y Pérez, G.A. (2009). Comparación de la efectividad de un insecticida botánico y dos químicos convencionales en el control del picudo (Anthonomus eugenii Cano) (Coleóptera: Curculionidae) en chile habanero (Capsicum chinense Jaqc.). Fitosanidad, 13(2), 117-120.

SAS Institute Inc. (2011). Base SAS ${ }^{\circledR} 9.3$ Procedures Guide: Statistical Procedures. Cary, NC: SAS Institute Inc.

Servín, R., García, H.J.L. y Troyo, D.E. (2007). Buenas prácticas en el manejo plagas para una agricultura, ganadería y producción forrajera sostenible en zonas áridas. Editorial Centro de Investigaciones Biológicas del Noroeste, S.C. Primera Edición. La Paz B.C.S. México. $85 \mathrm{p}$.

SIAP (2016). Servicio de Información Agroalimentaria y Pesquera, México. Anuario estadístico de la producción agrí́cola 2014. Recuperado el 11 de agosto de 2016, de www.siap.gob.mx/cierre-de-la-produccion-agricola-por-estado

Toapanta, M.A., Schuster, D.J. \& Stansly, P.A. (2005). Development and life history of Anthonomus eugenii (Coleoptera: Curculionidae) at constant temperatures. Environmental Entomology, 34(5), 999-1008. 
Whalon, M.E., Mota-Sánchez, D., Hollingworth, R.M. \& L. Duynslager (2008). Arthropod Pesticide Resistance Database. Michigan State University Extension. Recuperado el 03 de agosto de 2016, de wWw.pesticideresistance.org/ 